Einführung zum Thema

Unfallchirurg 2019 $122: 668-669$

https://doi.org/10.1007/s00113-019-0678-9

(c) Springer Medizin Verlag $\mathrm{GmbH}$, ein Teil von Springer Nature 2019

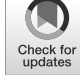

„Weg frei für die Telemedizin“,

so lautete die Schlagzeile des Beitrags im Deutschen Ärzteblatt vom Juni 2018, in dem über den Beschluss des Deutschen Ärztetages zur Lockerung des Fernbehandlungsverbotes berichtet wurde. Künftig sollen eine Beratung und Behandlung über Kommunikationsmedien auch ohne persönlichen Erstkontakt im Einzelfall erlaubt sein, ,wenn dieses ärztlich vertretbar ist und die erforderliche ärztliche Sorgfalt ... gewahrt wird." Mit dieser Änderung der ärztlichen Musterberufsordnung reagierte der Deutsche Ärztetag auf die rasche Verbreitung der Telemedizin in den letzten Jahren, die oft ohne Einflussmöglichkeit der Standesorganisationen stattfand. Seither ist die Diskussion um die Telemedizin als Teil der zunehmenden Digitalisierung des Gesundheitssystems deutlich wirkmächtiger geworden. Und wie immer, wenn es um die disruptiven Auswirkungen einer - in diesem Falle gar nicht so neuen - Technologie geht, halten sich Befürworter und Kritiker die Waage.

\section{》) Ist die Telemedizin der medizinische Standard der Zukunft?}

Die eher utopisch gestimmten Befürworter verorten die Telemedizin als einen Teil der digitalen Gesundheit in ein neues Zeitalter, in dem effiziente, benutzerfreundliche und umfassend akzeptierte elektronische Gesundheitsdienste die Qualität und Zugänglichkeit in der medizinischen Versorgung verbessern werden. Digital ist überall: Wearables und Apps übermitteln individuelle Ge-

Literatur beim Verfasser

\author{
W. Mutschler \\ Klinik für Allgemeine, Unfall-, und Wiederherstellungschirurgie, München, Deutschland
}

\title{
Weg frei für die Telemedizin
}

sundheits- und Krankheitsparameter; Chatbots wie AdaHealth ersetzen Dr. Google bei der Suche und der Anwendung von medizinischem Fachwissen, unterstützen Ärzte bei der Diagnosefindung und bieten Therapieempfehlungen an. In weiterem Rahmen analysieren auf künstlicher Intelligenz (KI) basierende Algorithmen große Datenmengen im Sinne von "deep learning“, verarbeiten z. B. laborchemische, histopathologische und Befunde aus der Bildgebung und führen diese dann zusammen. Man erhofft sich davon verlässlichere Diagnosen/ Differenzialdiagnosen und ausgeklügelte, evidenzbasierte Entscheidungshilfen. Manche Experten gehen inzwischen so weit zu postulieren, dass in einigen Jahren grundlegende Therapieentscheidungen nicht mehr ohne Gegenprüfen durch KIbasierte Programme getroffen werden (dürfen). Eine ausgebaute IT-Struktur vorausgesetzt, bieten KI-basierte Systeme künftig auch das Potenzial, nicht nur individuelle Behandlungsabläufe fundamental zu verändern, sondern darüber hinaus eine Lotsenfunktion im Gesundheitssystem zu übernehmen. So gesehen, birgt Medizin 4.0 zweifellos sehr große Möglichkeiten.

Und die Risiken? Kritiker sehen neben den Problemen einer ausufernden Datenflut, dem Datenschutz, der Haftung und der Vergütung v.a. zwei generell zu berücksichtigende Handlungsfelder.

Zum einen: Die Digitalisierung wird den Arzt nicht ersetzen können und wollen, aber sie wird die Formen der Gesundheitsleistungen dramatisch verändern. $\mathrm{Zu}$ hinterfragen sei deshalb, ob der Arzt zukünftig Steuermann oder Maschinist am Computer sei. Wie behalten Arzt und Patient die Datensouveränität und Deutungshoheit über ihre Daten? Wie entwickeln wir die notwendige di- gitale Gesundheitskompetenz („,e-health literacy") bei Patienten und Gesundheitsberufen gleichermaßen? Ist das Versprechen realistisch, dass digitale Entlastung und Unterstützung bei Ärzten und Pflegekräften mehr Raum und Zeit für den unmittelbaren, zuwendenden Dialog lassen?

Zum zweiten: Der globale digitale Gesundheitsmarkt wächst rasant, um ca. $20 \%$ jährlich. Immer mehr große, multinationale und v.a. kapitalorientierte Konzerne sehen das Marktvolumen. Welche Interessen werden sie letztlich vertreten, die der Eigentümer oder die der vielfältigen und nichtorganisierten Adressaten? Und wie lassen sich multinationale Aktivitäten durch nationale Gesetzgebungen und Regularien beeinflussen und kontrollieren?

Zurück zur Telemedizin. Die bekanntesten medizinischen Online-Beratungsdienste für Patienten sind im Ausland angesiedelt. Das schweizerische Unternehmen Medgate, mit dem Slogan "doc around the clock“ unterwegs, ist z. B. eines der größten telemedizinischen Zentren Europas und bietet eine arztbasierte allgemeine Beratung, Diagnostik, Rezeptierung und Überweisungen an. Als telemedizinischer Dienstleister gestartet, hat das Unternehmen inzwischen eigene Ärztezentren gegründet und ein Partnernetzwerk etabliert, dem Grundversorger, Spezialisten, Spitäler und Apotheken angehören. Gefordert durch die gesundheitspolitischen Vorgaben etwa im E-Health-Gesetz und die anstehende Einführung der elektronischen Gesundheitsakte entwickeln in Deutschland die gesetzlichen Krankenversicherungen und die privaten Krankenversicherungen ähnliche Vorhaben. In Baden-Württemberg testet die kassenärztliche Vereinigung mit DocDirekt 
derzeit ein Versorgungskonzept für die ambulante vertragsärztliche Versorgung.

Unabhängig davon gibt es in der Bundesrepublik Deutschland bereits seit vielen Jahren bestehende Initiativen zur poststationären Betreuung, in denen Patienten in häusliche telemedizinische Überwachungs- und Anleitungsprogramme eingebunden sind, z. B. durch Telemonitoring von Schrittmachern und Defibrillatoren, von Diabetes und COPD oder durch Telerehabilitation nach Schlaganfall. Auf der Arzt-ArztEbene seien hier stellvertretend Teledermatologie, Telepathologie und Teleradiologie genannt. Sie dienen nicht nur der Primärbefundung oder der interkollegialen Konsultation, sondern können auch zur Qualitätssicherung und für die Fort- und Weiterbildung etwa mit strukturierten Fallsammlungen genutzt werden. Andere Anwendungsgebiete sind die institutionalisierte Telemedizin, etwa die Unterstützung von Krankenhäusern der Maximalversorgung für kleinere Versorger. In Bayern z. B. sind in dem Netzwerk TEMPiS, telemedizinisches Projekt zur integrierten Schlaganfallversorgung, 21 regionale Kliniken in Südostbayern mit zwei überregionalen Schlagananfallzentren gekoppelt. Hier wird nicht nur innerhalb von Minuten über eine notwendige Lysetherapie vor Ort entschieden; die teilnehmenden Kliniken haben sich auch zur regelmäßigen Fortbildung und zur Eingabe ihrer Daten in ein Schlaganfallregister verpflichtet. Und viele Universitätskliniken haben begonnen, dem Vorbild des Uniklinikums Aachen folgend, regionale Netzwerke für eine Teleintensivmedizin aufzubauen.

\section{I) Telemedizin - eine Chance für akut und chronisch Kranke}

Begleitet werden diese und viele andere Modellprojekte von einer großen Anzahl neu gegründeter telemedizinischer Zentren, wissenschaftlicher Plattformen und den wissenschaftlichen Fachgesellschaften im Rahmen von Kongressbeiträgen, Fachartikeln, Studiengängen und von Förderungen durch das Bundesge- sundheits- und Bundesforschungsministerium sowie Förderprogrammen der Bundesländer.

Es ist also viel in Bewegung, auch in unserem Fach O\&U. Einige der Aktivitäten wollen wir in unserem Leitthema zur Telemedizin vorstellen. Es beginnt mit Prof. Siegfried Jedamzik, einem Pionier der Telemedizin. Er gibt zunächst eine allgemeine Einführung in die Telemedizin, schildert dann drei konkrete Vorhaben, die telefonisch-medizinische Notfalltriage GOINakut, die Online-Videosprechstunde Doccura und die digitale Pflegeakte „Speed“ und schließt mit einem faszinierenden Ausblick in die digitale Medizin von morgen. Markus Blätzinger, Geschäftsführer der Akademie der Unfallchirurgie, stellt dann die von Prof. Johannes Sturm initiierte Telekooperationsplattform TKmed im Traumanetzwerk DGU im Detail vor. Mit über 700 Anwendern und mehr als 4 Mio. übertragenen Bilddaten/Monat gehört TKmed zu den größten deutschen Netzwerken im akutklinischen Bereich. Dr. Stephan Prückner und seine Arbeitsgruppe am Institut für Notfallmedizin und Medizinmanagement der LMU München beschäftigen sich mit den Zielen, Voraussetzungen und Anforderungen sowie der praktischen Umsetzung und den Einsatzmöglichkeiten für ein Telenotarztsystem. Eine Bestandsaufnahme von Dr. Florian Dittrich et al. zum klinischen Einsatz mobiler Apps in Orthopädie und Unfallchirurgie rundet das Themenheft ab.

Noch steht die Telemedizin als Teil von E-Health in unserem Fachgebiet sicherlich am Anfang. Dass digitale Technologien in Zukunft auch O\&U prägen werden, ist eine Binsenwahrheit. Dass sie, richtig eingesetzt, schon jetzt zur Verbesserung der Kommunikation PatientArzt, Arzt-Arzt und zwischen medizinischen Institutionen und dort gerade an sektoralen Schnittstellen beitragen und so die Struktur- und Prozessqualität erhöhen, ist gut belegt. Ob die Ergebnisqualität mit der Telemedizin signifikant gesteigert werden kann, ist derzeit Gegenstand vielfältiger Evaluationen.

Wir als Herausgeber von Der Unfallchirurg bleiben an dem Thema dran und hoffen, auch bei unseren Lesern die Diskussion anzustoßen.

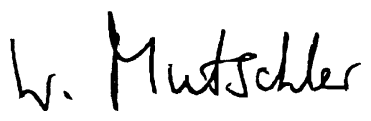

Prof. Dr. Wolf Mutschler, München

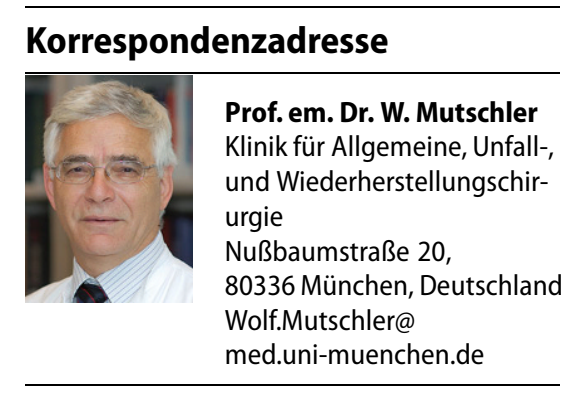

Interessenkonflikt. W. Mutschler gibt an, dass kein Interessenkonflikt besteht. 